\title{
Design of a new hybrid artificial neural network method based on decision trees for calculating the Froude number in rigid rectangular channels
}

\author{
Isa Ebtehaj $^{1,2}$, Hossein Bonakdari ${ }^{1,2^{*}}$, Amir Hossein Zaji ${ }^{1,2}$, Charles Hin Joo Bong ${ }^{3,4}$, \\ Aminuddin Ab Ghani ${ }^{4}$ \\ ${ }^{1}$ Department of Civil Engineering, Razi University, 67149-67346 Baghe Abrisham, Kermanshah, Iran. \\ ${ }^{2}$ Water and Wastewater Research Center, Razi University, 67149-67346 Baghe Abrisham, Kermanshah, Iran. \\ ${ }^{3}$ Department of Civil Engineering, Faculty of Engineering, University Malaysia Sarawak, 94300 Kota Samarahan, Sarawak, Malaysia. \\ ${ }^{4}$ River Engineering and Urban Drainage Research Centre (REDAC), Universiti Sains Malaysia (USM), 14300 Nibong Tebal, \\ Pulau Pinang, Malaysia. \\ * Corresponding author. Tel.: +98 833427 4537. Fax: +98 833428 3264. E-mail: bonakdari@yahoo.com
}

\begin{abstract}
A vital topic regarding the optimum and economical design of rigid boundary open channels such as sewers and drainage systems is determining the movement of sediment particles. In this study, the incipient motion of sediment is estimated using three datasets from literature, including a wide range of hydraulic parameters. Because existing equations do not consider the effect of sediment bed thickness on incipient motion estimation, this parameter is applied in this study along with the multilayer perceptron (MLP), a hybrid method based on decision trees (DT) (MLP-DT), to estimate incipient motion. According to a comparison with the observed experimental outcome, the proposed method performs well $(M A R E=0.048, R M S E=0.134, S I=0.06, B I A S=-0.036)$. The performance of MLP and MLP-DT is compared with that of existing regression-based equations, and significantly higher performance over existing models is observed. Finally, an explicit expression for practical engineering is also provided.
\end{abstract}

Keywords: Decision tree; Incipient motion; Multilayer perceptron (MLP); Froude number.

\section{INTRODUCTION}

Sediment deposition on the beds of rigid boundary channels, such as sewer, drainage and irrigation channels is correlated to extensive economic and technical problems. Deposits on channel beds lead to roughness, and in contrast to smooth beds, hydraulic behavior and performance can be significantly altered. Therefore, accurately predicting and determining sediment incipient motion is noteworthy in the optimum design of transport systems.

Minimum velocity and shear stress are among the most effective factors in reducing sedimentation, and different criteria for both shear stress and velocity were provided by Vongvisessomjai et al. (2010). This method does not refer to sediment and flow characteristics and is thus not reliable, as it leads to under or overestimated critical velocity for incipient motion (Ebtehaj et al., 2014). Therefore, several experimental research works have been conducted with a large variety of equations to estimate the incipient motion velocity ( $\mathrm{Ab}$ Ghani et al., 1999; El-Zaemey, 1991; Novak and Nalluri, 1984; Safari et al., 2011). Some equations are applied extensively in many designs owing to their simplicity, but these equations do not consider the thickness of sediment deposited on the bed, which has significant impact on the dimensionless shear stress in the Shields diagram (Bong et al., 2013). Moreover, since the equations are regression-based their performance in different hydraulic conditions is questionable.

Due to the ability of artificial neural networks (ANNs) to solve complex problems and the good performance in different engineering sciences, this method is often used in hydraulic and environmental engineering ( $\mathrm{Ab}$ Ghani et al., 2011; Ahmad et al., 2011; Azamathulla et al., 2008; Ebtehaj and Bonakdari, 2016; Haddadchi et al., 2013). To predict the minimum sediment transport velocity in sewers, Ebtehaj and Bonakdari (2013) utilized an MLP neural network. The results indicated that the MLP neural network can highly accurately estimate the minimum velocity. Furthermore, a comparison between the MLP neural network and traditional equations demonstrated the superior performance of the MLP neural network. Sun et al. (2014) investigated the capability of ANN to predict the velocity distribution in combined open channels using computational fluid dynamics data. Kizilöz et al. (2015) predicted scour around submarine pipelines using ANN and found that the ANN results are in good agreement with the measured data.

To enhance intelligent method performance hybrid methods are widely used, for instance the Taguchi-genetic algorithm for adaptive network-based fuzzy inference system training (Ho et al., 2009); the hybrid group method of data handling (GMDH) with genetic programming (Najafzadeh and Barani, 2011); evolutionary algorithms to optimize ANN layer weights (Ebtehaj and Bonakdari, 2014); and a combined firefly algorithm (FFA) and wavelet with support vector machines (SVM), i.e. SVM-FFA and SVM-Wavelet (Gocić et al., 2015). Kavousi-Fard and Kavousi-Fard (2013) used a new hybrid method of the cuckoo search algorithm (CSA), autoregressive integrated moving average (ARIMA) and support vector regression (SVR) for short-term load forecasting problems. Najafzadeh and Lim (2014) developed a neuro fuzzy-based GMDH method using the particle swarm optimization (PSO) learning algorithm (NFGMDH-PSO) to predict scour downstream of a sluice gate. The authors found that the proposed method performed better than traditional methods. Bonakdari and Ebtehaj (2014) used the imperialist competitive algorithm (ICA) to improve multilayer perceptron (MLP) neural network application in predicting sediment transport at limit of deposition. A comparison between MLP and MLP-ICA indicated the significant increase in radial basis function (RBF) capability when using MLP as an evolutionary learning algorithm.

The main aim of this study is to develop the MLP neural network using decision trees (DT) for predicting the incipient 
sediment motion in rigid boundary rectangular stormwater channels by using three experimental datasets (Bong et al., 2013; Salem, 2013; Shvidchenko and Pender, 2000). The results of the MLP neural network and several regression-based equations are compared with the MLP-DT results.

\section{OVERVIEW OF EXITING EQUATIONS}

Novak and Nalluri (1984) conducted different experimental tests on the incipient motion of sediment particles in flumes with rough and smooth beds. The flumes were rectangular glass-walled, $15 \mathrm{~m}$ and $6 \mathrm{~m}$ long and $300 \mathrm{~mm}$ wide. The median diameters of sediment particles ranged from $3.6 \mathrm{~mm}$ to 37.2 $\mathrm{mm}$. Novak and Nalluri (1984) considered $\mathrm{Fr}$ as a function of $d / R$ and presented the following equation:

$F r=0.5\left(\frac{d}{R}\right)^{-0.5}$

where $F r$ is the densimetric Froude number $\left(=V_{c} /(g(s-1) d)^{0.5}\right)$, $V_{c}$ is the critical velocity, $d$ is the median diameter of particles, $g$ is the gravitational acceleration, $s$ is the specific gravity of sediment $\left(=\rho_{s} / \rho\right), \rho$ and $\rho_{s}$ are the fluid and sediment density (respectively) and $R$ is the hydraulic radius.

In order to present an equation for estimating the critical velocity $\left(V_{c}\right)$ of the incipient motion of sediment, El-Zaemey (1991) performed different experimental tests with sediments ranging from 2.9 to $8.4 \mathrm{~mm}$. The maximum diameter of sediment particles employed was greater than Novak and Nalluri's (1984). El-Zaemey (1991) presented the following equation, which has the same form as Novak and Nalluri's (1984) equation but different coefficients:

$F r=0.75\left(\frac{d}{R}\right)^{-0.34}$

To investigate the effect of sediment deposit thickness on the incipient motion of sediment, Ab Ghani et al. (1999) conducted tests in a rigid rectangular channel $10 \mathrm{~m} \times 300 \mathrm{~mm} \times 450 \mathrm{~mm}$ $(L, W, D)$. The range of sediment particles used was $0.55<d$ $(\mathrm{mm})<4.78$. The authors evaluated the available criteria to survey the performance on a bed with sediment deposits. It was found that existing equations cannot consider the effect of sediment bed thickness. Therefore, they applied sediment bed thickness $\left(t_{s}\right)$ of $d<t_{s}(\mathrm{~mm})<10$ in the tests and produced the following equation:

$$
F r=1.07\left(\frac{d}{R}\right)^{-0.23}
$$

Safari et al. (2011) presented an equation to estimate the incipient motion of sediment in rigid boundary channels with rectangular and V-shape bottom cross-sectional areas using two different datasets. The range of the median diameter of particles was $0.87<d(\mathrm{~mm})<7.72$ and the specific gravity was 2.65 . The best fit equation presented by the authors is as follows:

$F r=0.68\left(\frac{d}{R}\right)^{-0.41}$

Salem (2013) carried out a series of tests on a rectangular flume to survey the incipient motion of sediment. The range of particle sizes in the experimental tests was $0.55<d(\mathrm{~mm})<$ 4.78. The author presented an equation in two forms. The first equation is the same as the equations presented earlier (by $\mathrm{Ab}$ Ghani et al., 1999; El-Zaemey, 1991; Novak and Nalluri, 1984) in the form of $F r=a(d / R)^{b}$ (Equation 5), and the second is inspired from Ackers and White's (1973) equation (Equation 6). Salem's (2013) equations are as follows:

$$
\begin{aligned}
& F r=0.937\left(\frac{d}{R}\right)^{-0.255} \\
& F r=-0.523 \ln \left(\frac{d}{R}\right)+0.476
\end{aligned}
$$

Bong et al. (2013) performed experiments in a rectangular channel keeping in view the effect of sediment deposit thickness. The median diameter of sediment $(d)$ and specific gravity of sediment were $0.81<d(\mathrm{~mm})<4.78$ and $2.5<s<2.57$ respectively. The authors deemed sediment deposit thickness $\left(t_{s}\right)$ to be an effective parameter in estimating the critical velocity of incipient motion. Their equation is as follows:

$F r=1.17\left(\frac{d}{R}\right)^{-0.167}\left(\frac{t_{s}}{d}\right)^{0.0378}$

where $t_{s}$ is the sediment deposit thickness.

\section{MATERIALS AND METHODS Data collection}

In this study, Bong et al. (2013), Salem's (2013) and Shvidchenko and Pender (2000) datasets are used for estimating the $F r$. Shvidchenko and Pender (2000) conducted experiments in a rectangular, glass-walled channel $8 \mathrm{~m} \times 0.3 \mathrm{~m} \times 0.3 \mathrm{~m}(L, W$, $D$ ). The maximum discharge produced by the pump was $30 \mathrm{~L} / \mathrm{s}$. The flow regime was controlled using a tailgate located at the output of the channel. Uniform bed thickness of $5 \mathrm{~cm}$ was intended for situations in which the total length of the sediment bed was $5.6 \mathrm{~m}$. The slope of the flume used in all of Shvidchenko and Pender's (2000) tests ranged from 0.0014 to 0.02870 . The median diameter of particles was $1.5-4.5 \mathrm{~mm}$.

Bong et al. (2013) carried out experiments on a rectangular channel. The discharge and flow velocity values were measured using an electromagnetic current meter. This current meter was verified using the calculated discharge of a V-notch weir installed downstream of the channel. Bong et al. (2013) did tests with 4 slopes (i.e. $1 / n ; n=200,350,500,1000$ ). The thickness of the sediments used in the tests was such that first, a layer of sediment was placed on the bed $\left(t_{s}=d\right)$, after which the thickness of sediment on the channel bed was increased $\left(t_{s}=5,10\right.$, $24 \mathrm{~mm}$ ). Bong et al. (2013) selected sediment size and thickness on the channel bed slope based on field observations by Ab Ghani et al. (2001). The total thickness of the sediment bed was $2.1 \mathrm{~m}$. The observation section was $3.5 \mathrm{~m}$ from upstream, where according to initial observations the flow was fully developed.

Salem (2013) conducted experimental tests in a rectangular channel $10 \mathrm{~m} \times 450 \mathrm{~mm} \times 300 \mathrm{~mm}(L, W, D)$. The side walls of the flume were made of glass so the particles' movement on the bed would be visible. The depth of flow in the flume was set with a tail gate. The horizontal slope of the channel was set with a mechanical jack placed near the channel output. While 
increasing the velocity until attaining the required velocity that led to the initial movement of sediments (incipient motion), the flow depth, flume slope and discharge values were recorded. The minimum thickness of the sediment bed in Salem's (2013) tests was $d$. Thus, the sediment bed was spread over the entire channel width. The hydraulic ranges and sediment characteristics are presented in Table 1.

\section{Dimensional analysis}

The components required to define the three-phase flow of air, water and sediment include flow, fluid and sediment characteristics (Yalin, 1977). In providing a functional equation to estimate the critical velocity $\left(V_{c}\right)$, it is recommended to consider fluid characteristics (such as fluid density $(\rho)$ ), sediment features (such as density $\left(\rho_{s}\right)$, median diameter of particles $(d)$ ) and flow characteristics (such as gravity acceleration $(g)$ and flow depth or hydraulic radius $(R)$ ). In addition to the above parameters, Bong et al. (2013) suggested defining the $t_{s}$ parameter so that the sediment bed thickness would be considered an effective parameter in determining the incipient sediment motion. Therefore, the parameters affecting minimum velocity calculation are given in the following functional relationship:

$$
V_{c}=\Phi\left(\rho, \rho_{s}, d, R, g, t_{s}\right)
$$

Literature studies indicate that the initial velocity is the Froude number dimensionless parameter $\left(F r=V /(g(s-1) d)^{0.5}\right)$ and dimensional analysis suggests the following equation to calculate the $\mathrm{Fr}$ parameter:

$$
F r=\frac{V_{c}}{\sqrt{g d(s-1)}}=f\left(\frac{d}{R}, \frac{t_{s}}{R}\right)
$$

where $F r$ is the densimetric Froude number, $V_{c}$ is the critical velocity, $g$ is the gravitational acceleration, $d$ is the median diameter of particles, $s$ is the specific gravity of sediment $\left(=\rho_{s} / \rho\right), R$ is the hydraulic radius and $t_{s}$ is the sediment bed thickness.

A hybrid multilayer perceptron (MLP) method based on decision trees (DT) (MLP-DT) is used to estimate Fr. Therefore, the datasets should be classified into two categories: training and testing. Among 235 existing data, random sampling without replacement was used to obtain 70 data $(30 \%)$ for model validation and $165(70 \%)$ for model training. Subsequent to estimation and performance confirmation in both training and testing, the estimation accuracy of this model was compared with existing equations.

\section{Multi-layer perceptron neural network}

The MLP neural network is successfully used in different engineering fields to simulate complex problems (Haykin,
1994). A typical MLP (Figure 1) has three types of layers: input, hidden and output layers (Hagan et al., 1996; Haykin, 1994; Oliver, 2001). The initial information of the problem is submitted to the MLP neural network via the input layer. The input layer's number of neurons is equal to the number of input variables of the considered problem. Each neuron in the input layer is connected to a neuron in the hidden layer. In the hidden layer, a nonlinear transformation is done to the received information (Christopher, 1995) using activation functions. In this study, the sigmoid activation function is used for the hidden layer as shown in Eq. (10).

$$
f(x)=\frac{2}{1+e^{-2 x}}-1
$$

Subsequently, the output layer result is prepared by multiplying the weighting factor of the hidden layer's information, as presented in Eq. (11).

$$
\text { Neuron }_{j}=\sum_{i=1}^{n} W_{i j} F_{i}
$$

where Neuron ${ }_{j}$ is the network process result; $W_{i j}$ are the connecting weights between the hidden layer neurons and output layer neurons, respectively; and $F_{i}$ is the hidden layer's neuron information that is transferred to the next layer. As such, the output layer acts like a linear regressor of the hidden layer neurons' information. The output layer results are the MLP neural network outcomes that can be compared with the observed or experimental data. According to Eq. (11), the numbers of input and output layer neurons are equal to the numbers of input and output variables of the considered problem. However, there is no definitive rule to determine the number of hidden layer neurons. In this study, trial and error is used to determine the number of hidden layer neurons in the MLP models employed (Bilhan et al., 2010; Breiman et al., 1993; Kisi, 2008).

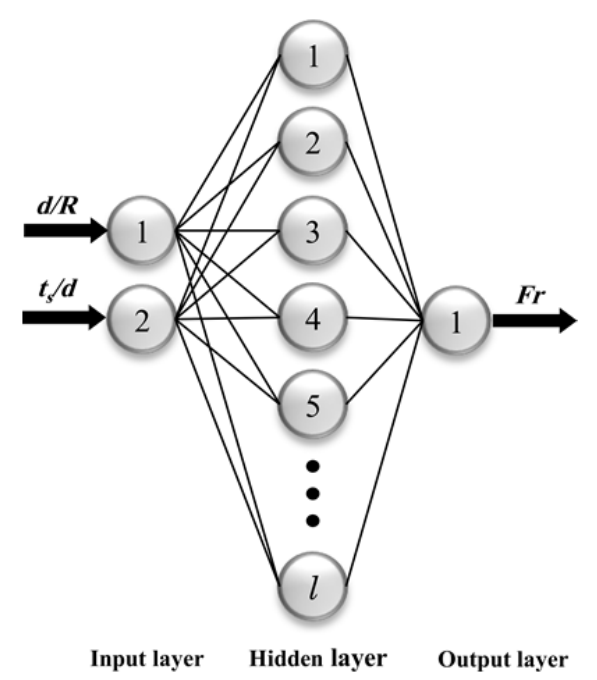

Fig. 1. MLP structure used for the Incipient Motion problem.

Table 1. Dataset ranges.

\begin{tabular}{lcccccc}
\hline Authors & $L, W, D(\mathrm{~m})$ & $s=\left(\rho_{s} / \rho\right)$ & $d(\mathrm{~mm})$ & $t_{s}(\mathrm{~mm})$ & $y(\mathrm{~m})$ & $V_{c}(\mathrm{~m} / \mathrm{s})$ \\
\hline Salem (2013) & $10,0.3,0.45$ & $2.3-2.57$ & $0.5-4.78$ & $0.5-24$ & $0.0135-0.159$ & $0.188-0.619$ \\
Bong et al. (2013) & $6,0.6,0.4$ & $2.54-2.57$ & $0.81-4.78$ & $0.81-24$ & $0.007-0.132$ & $0.216-0.442$ \\
Shvidchenko and Pender (2000) & $8,0.3,0.3$ & $2.6-2.65$ & $1.5-4.5$ & $50-50$ & $0.015-0.136$ & $0.065-0.865$ \\
\hline
\end{tabular}




\section{Hybrid decision tree-based multilayer perceptron neural network}

MLP-DT, a novel, hybrid artificial neural network is introduced in this section. In this method, DT classification (Breiman et al., 1993) is used to attain a regression method with higher performance. The classification part of MLP-DT serves to predict the class of each sample using the input variables of the considered problem. In the DT process, recursive splitting is done to find the best divisions of input variables. First, the dataset is divided into two classes by placing a partition on one of the input variables. This partition is placed by trial and error. It means the whole dataset is divided in various places of each one of the input variables to determine which one leads to the highest classification performance. Subsequently, the next split is found through the same process. Splitting is done repetitively to find the best DT for the considered problem. In the present study, DT training stops if all tree leafs reach the considered Minimum Parent Size (MPS). Thus, after each partitioning, the number of each leaf's samples is checked. If the number of leaf samples is higher than the MPS, the leaf is considered a branch and gets split again; else, the considered leaf is removed from the splitting process.

Then the DT divides the simple MLP neural network into smaller networks so that each small MLP simulates every part of the divided dataset. Figure 2 presents the MLP-DT method proceeding. According to the figure, in the first step the DT is trained to divide the whole dataset into classes by using the considered input and output variables. It should be noted that precise DT classification may cause overtraining, which may decrease regression performance. Thus, the goal of DT is not to obtain a division with the highest classification quality but rather, to attain a classification that leads to the highest MLP-DT model regression performance. However, highly misclassified samples of a weak DT classification model may decrease MLP-DT regression performance. Therefore, the trial and error method is utilized in the current study to identify the most appropriate DT classification performance that would lead to the highest MLP-DT regression model precision. As mentioned before, DT accuracy is controlled by the MPS. If the MPS is assumed to equal one, the DT becomes an exact classifier. For the present dataset, the MLP-DT with MPS of 15 performed the best. In the second step of the MLP-DT procedure, the MLP is split into the considered number of DT algorithm classes. In the present study, the DT classification process splits the entire dataset into three classes: "Low", "Med" and "High." Therefore, the MLP neural network is split into three smaller MLPs. In this way, to achieve a fair comparison between the formal MLP and MLP-DT methods, the maximum allowable number of hidden layer neurons in the MLP is considered equal to the number in the smaller MLPs of the MLP-DT model.

The final MLP-DT step is to collect the separate class results of each smaller MLP to generate the final model outcomes.

\section{Goodness of fit}

To evaluate the MLP and MLP-DT methods with the observed experimental values and compare those with existing regression-based equations, the following statistical indexes are utilized: root mean square error ( $R M S E)$, mean absolute relative error $(M A R E)$, scatter index $(S I)$ and BIAS.

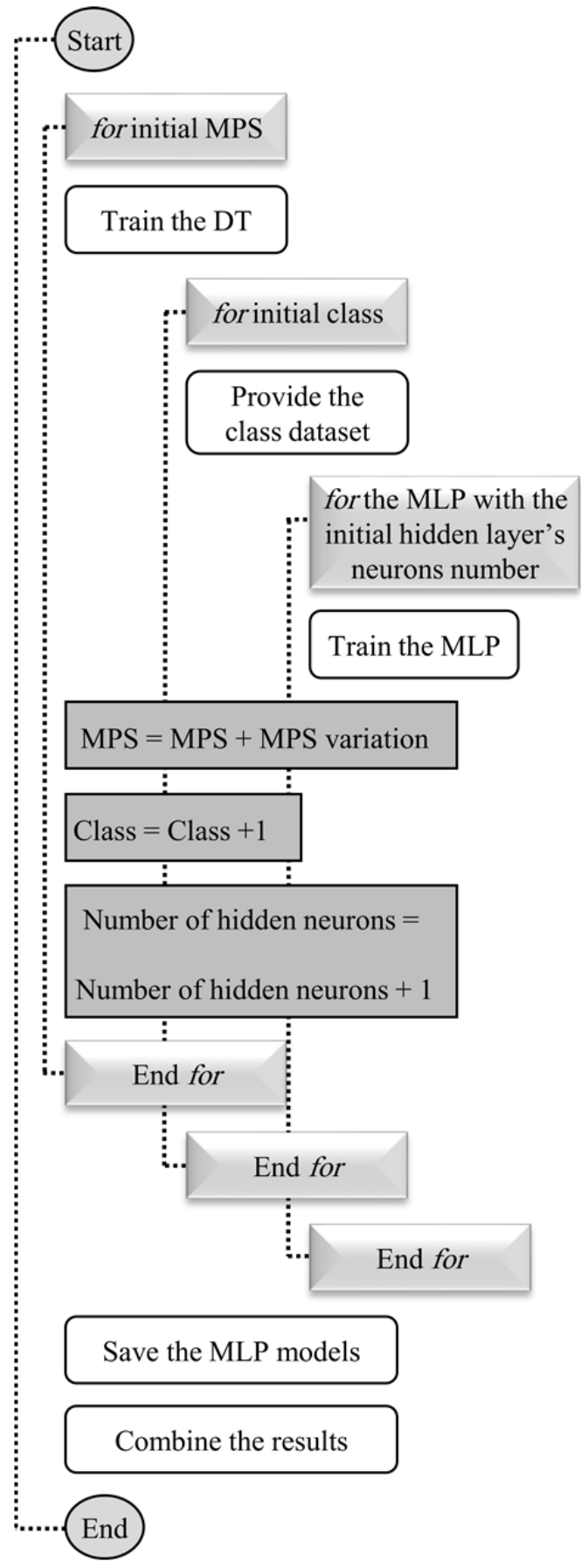

Fig. 2. DT procedure.

$R M S E=\sqrt{\frac{1}{n} \sum_{i=1}^{n}\left(F r_{\text {Observed } i}-F r_{\text {Predicted } i}\right)^{2}}$

MARE $=\frac{1}{n} \sum_{i=1}^{n} \frac{\left|F r_{\text {Observed } i}-F r_{\text {Predicted } i}\right|}{F r_{\text {Observed } i}}$

$S I=\frac{R M S E}{\overline{F r_{\text {Observed }}}}$

$B I A S=\frac{1}{n} \sum_{i=1}^{n}\left(F r_{\text {Predicted } i}-F r_{\text {Observed } i}\right)$ 
These indexes only exhibit the difference between the experimental and predicted values but not the forecast error distribution using MLP and MLP-DT; therefore, other indexes including absolute relative error (ARE) and threshold statistics (TS) are employed (Ebtehaj et al., 2015). The $T S_{x}$ error distribution index represents the predicted values for $x \%$ of the anticipated data. This index represents the ratio of predicted values with $A R E$ of less than $x \%\left(Y_{x}\right)$ to the total value.

$$
\begin{aligned}
& T S_{x}=\frac{Y_{x}}{n} \times 100 \\
& A R E=\frac{\left|F r_{\text {Observed }}-F r_{\text {Predicted }}\right|}{F r_{\text {Observed }}}
\end{aligned}
$$

\section{RESULTS AND DISCUSSION}

The results from simulating the incipient motion of sediment as the dimensionless parameter $F r$ using both MLP and MLP-DT are presented in this section. A comparison of the two models is shown in Figure 3. By considering the fact that the data employed has no significant role, MLP performed well in training and the relative error was slightly more than $10 \%$, indicating good training accuracy. However, MLP did not perform the same in model training and testing. Also according to Figure 3, the MLP simulation error in the testing phase significantly increased. Therefore, a method is still required that is flexible in situations with no significant effect on training. A hybrid decision tree (DT) and MLP were therefore applied to resolve this issue.

Figure 3 indicates that the MLP-DT model performed relatively comparable in both training and testing. In both cases, the values estimated by the proposed method had less than $10 \%$ relative error and data changes than in model training, signifying no significant impact on model performance.

Figure 4 shows the performance of existing regression-based equations in predicting the incipient motion of sediment. All equations use the dimensionless ratio of median particle diameter to hydraulic radius of flow area $(d / R)$ to estimate $F r$, except Bong et al.'s (2013) equation that uses the ratio of sediment bed thickness to the median particle diameter $\left(t_{s} / d\right)$. The difference between the two equations proposed by Salem (2013) is parameter $(d / R)$.
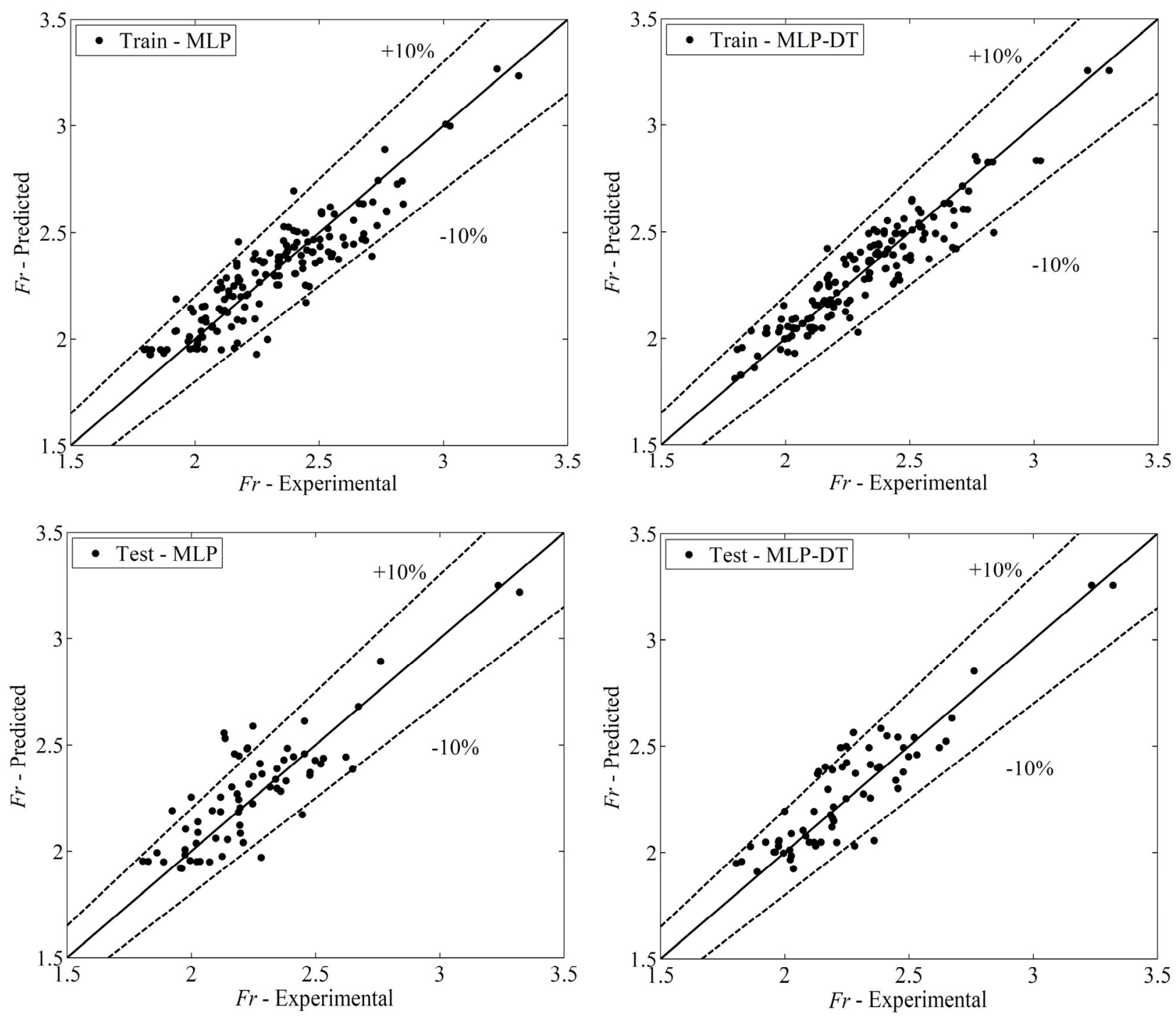

Fig. 3. Comparison of MLP and MLP-DT in predicting the $F r$ (training and testing). 


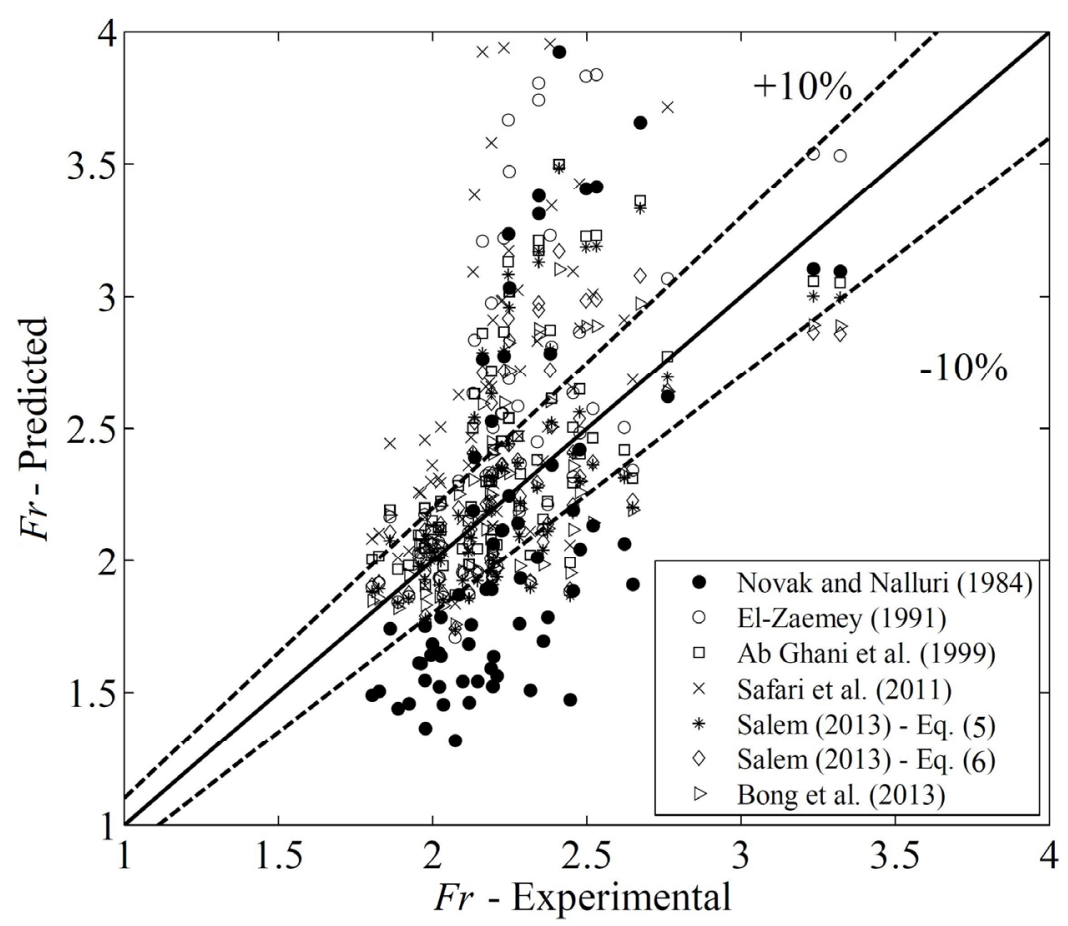

Fig. 4. Evaluation of existing regression-based equations in $\mathrm{Fr}$ prediction.

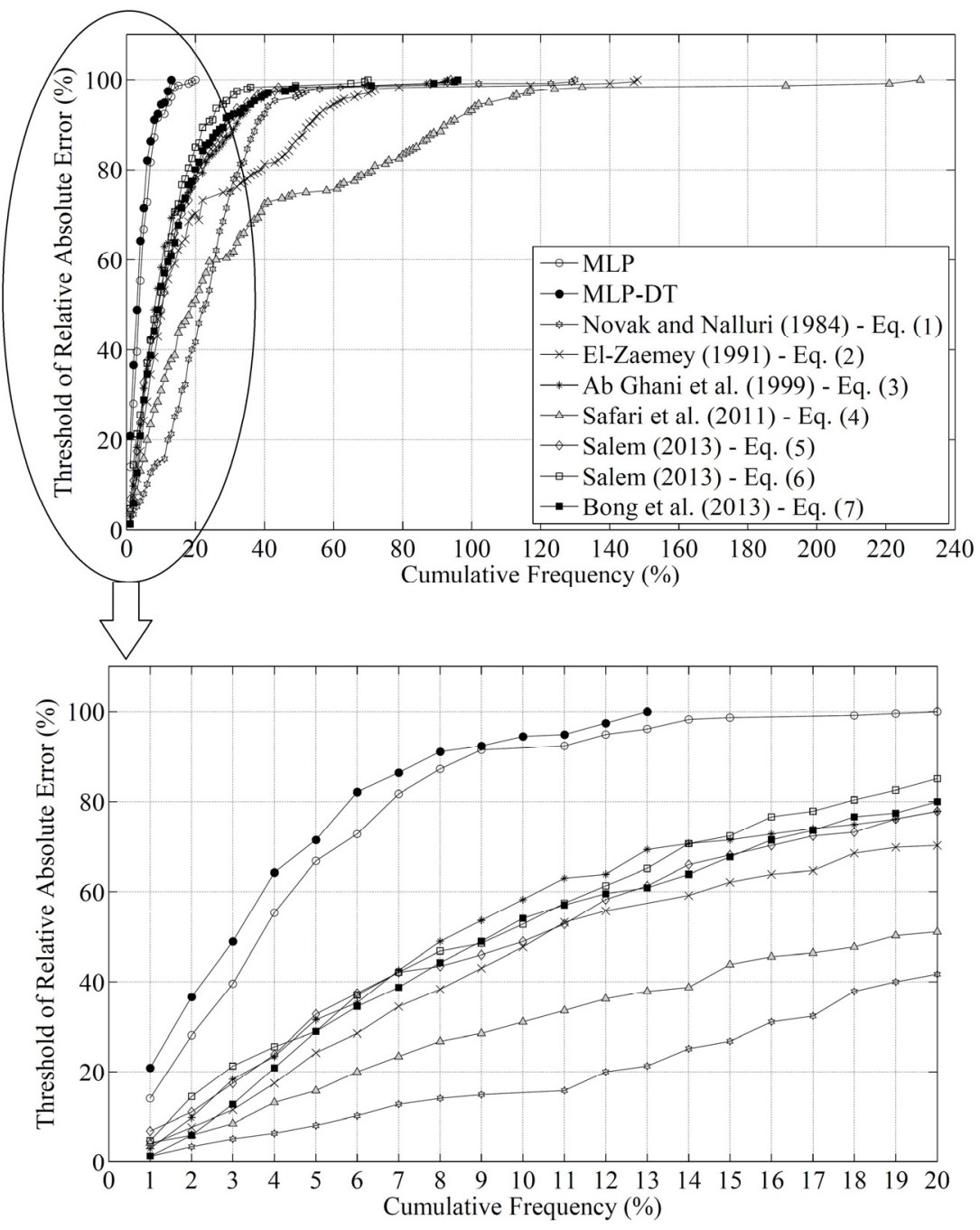

Fig. 5. Error distribution of MLP, MLP-DT and existing regression-based equations. 
In general, regression-based equations represent relatively poor performance and high error with various model predictions. Novak and Nalluri's (1984) equation includes estimations with large differences as under and overestimations. The statistical error indexes of the proposed models are presented in Table 2. According to this table, on average, Novak and Nalluri's (1984) equation overestimates $(B I A S=0.173)$. Therefore, it can be concluded that the overestimation values present greater error than the underestimation. According to Figure 5, the maximum error estimated by Novak and Nalluri's (1984) equation was about $140 \%$. Also, only $15 \%$ of estimations by this model show relative error below 10\%. El-Zaemey's (1991) equation did not perform well as it overestimated the $\mathrm{Fr}$ with about $16 \%$ mean relative error $(M A R E=0.167)$ and was three times greater than with MLP-DT and MLP. Figure 5 signifies that less than $50 \%$ of estimates made by this equation had a relative error below $10 \%$ while the maximum relative error of ML-DT was about 13\%. Ab Ghani et al.'s (1999) equation was similar to ML-DT, as it did not predict the incipient motion of sediment accurately. However, unlike the two previous equations, it underestimated $B I A S=-0.153$ on average and was more accurate than the equations of Novak and Nalluri (1984) and El-Zaemey (1991) $(M A R E=0.114, R M S E=0.358, S I=$ 0.159). Safari et al.'s (2011) new proposed equation to calculate the $\mathrm{Fr}$ had the weakest performance among all traditional equations $(M A R E=0.306, R M S E=0.554, S I=0.246, B I A S=$ $-0.68)$. The equation corresponds to an average relative error of about $30 \%$ and it underestimated the $F r$ by about 3 times the actual value (the maximum estimated value error was about 230\%) (Figure 5).

Table 2. Performance evaluation of MLP, MLP-DT and existing regression-based equations in predicting the incipient motion of sediment.

\begin{tabular}{|c|c|c|c|c|}
\hline Methods & $M A R E$ & RMSE & $S I$ & $B I A S$ \\
\hline MLP & 0.053 & 0.152 & 0.067 & -0.033 \\
\hline MLP-DT & 0.048 & 0.134 & 0.060 & -0.036 \\
\hline Novak and Nalluri (1984) - Eq. (1) & 0.208 & 0.548 & 0.244 & 0.173 \\
\hline El-Zaemey (1991) - Eq. (2) & 0.167 & 0.583 & 0.259 & -0.253 \\
\hline Ab Ghani et al. (1999) - Eq. (3) & 0.114 & 0.358 & 0.159 & -0.153 \\
\hline Safari et al. (2011) - Eq. (4) & 0.306 & 0.554 & 0.246 & -0.680 \\
\hline Salem (2013) - Eq. (5) & 0.109 & 0.349 & 0.155 & -0.051 \\
\hline Bong et al. (2013) - Eq. (7) & 0.096 & 0.269 & 0.120 & 0.008 \\
\hline
\end{tabular}

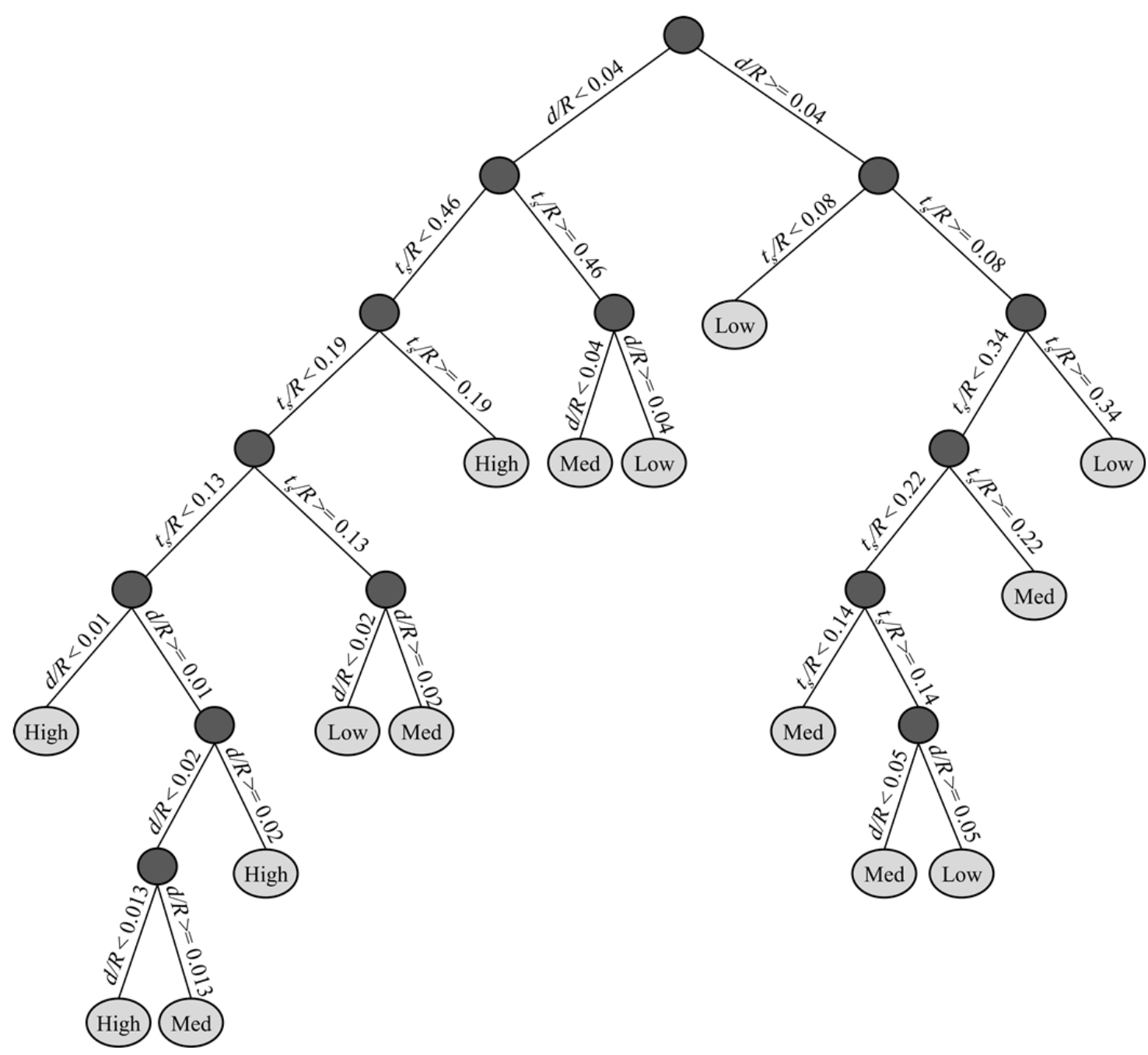

Fig. 6. DT for the incipient motion problem. 


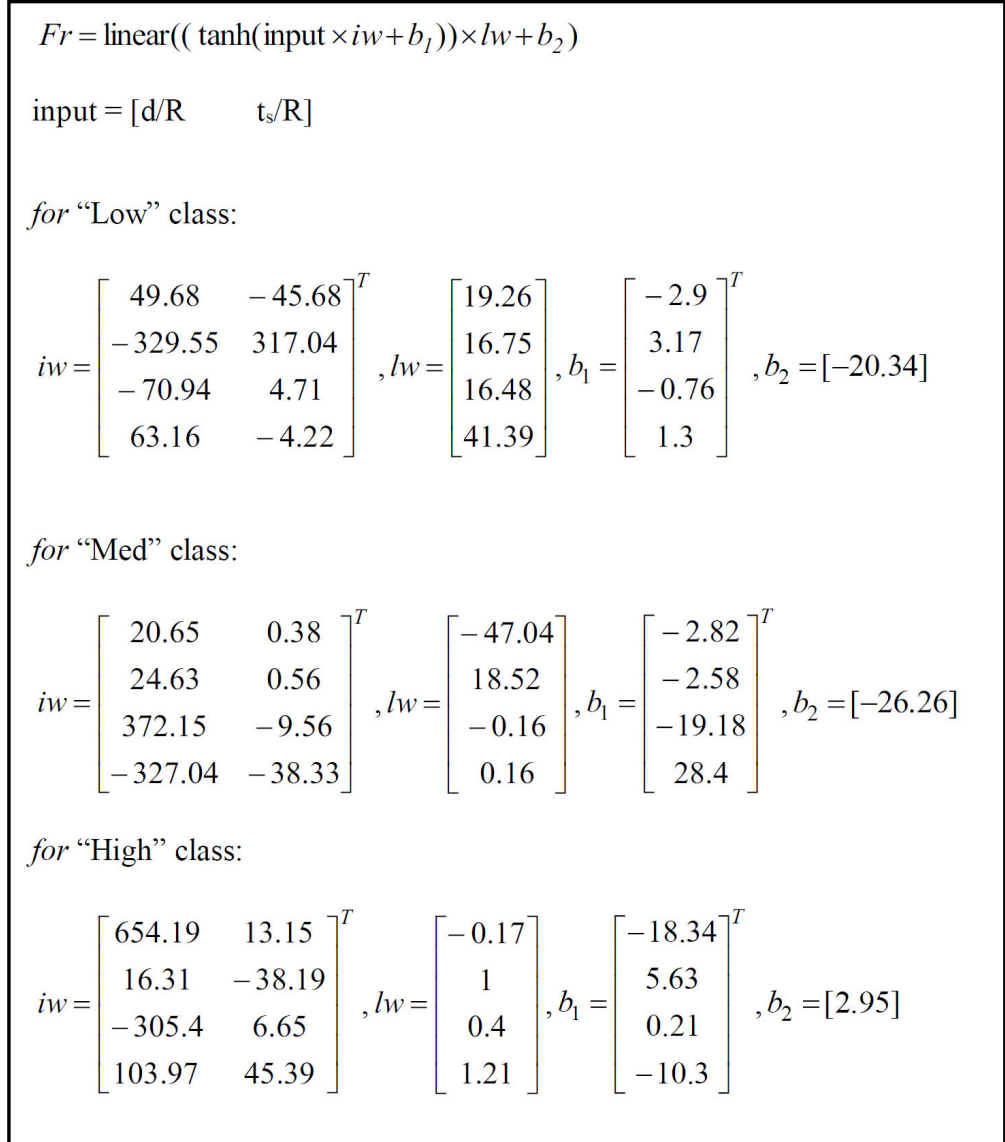

Fig. 7. MLP-DT equation for the incipient motion problem.

Eq. (8) presented by Salem (2013) solves the problems caused by the poor performance of other equations that use parameter $d / R$ to estimate $\operatorname{Fr}(M A R E=0.098, R M S E=0.2992, S I=0.13$, $B I A S=-0.036)$. However, it is observed that some of the presented estimates had large relative errors that sometimes reached more than $70 \%$. Bong et al. (2013) used the parameter of ratio of sediment bed thickness to the median diameter of particles $\left(t_{s} / d\right)$ and provided an equation that performs well in $\mathrm{Fr}$ estimation. Although the equation benefits from higher estimation performance, even compared with Salem's (2013) equation that is the best among those presented in the form of $F r=\Phi(d / R)$, it exhibited superior performance $(M A R E=0.096$, $R M S E=0.269, S I=0.12, B I A S=0.008)$. However, Bong et al.'s equation showed only about $55 \%$ of estimations with relative error below $10 \%$ and maximum relative error of more than $50 \%$. Meanwhile, MLP-DT with $92 \%$ estimation of all data can solve regression-based problems with relative error of less than $10 \%$ and maximum relative error of about $13 \%$. Figure 6 presents the final DT for the incipient motion problem.

According to the tree in Figure (6), the explicit equation using MLP-DT to calculate $F r$ is given in the Figure 7.

From Figure (7), it is obvious that the sum of the number of hidden layer neurons in the MLPs employed in the MLP-DT model is 12 .

\section{CONCLUSIONS}

Due to the extreme importance of determining the incipient motion of sediment for rigid boundary channel design, the critical velocity for incipient motion was estimated in this study using both the multilayer perceptron (MLP) neural network and a hybrid method of MLP based on decision trees (DT) (MLP-DT). The methods presented in this study performed well in $\mathrm{Fr}$ estimation, both with a mean relative error of about $5 \%($ MARE $(\mathrm{MLP})=0.053$, MARE $(\mathrm{MLP}-\mathrm{DT})=0.048)$. The hybrid method proposed (MLP-DT) can reduce the MLP's lack of flexibility and high-error estimation in different modes. As a result, the highest relative error achieved was about 13\%. An explicit equation to calculate the $\mathrm{Fr}$ was also provided. Existing equations indicate that all methods perform poorly compared to the model proposed in this study. The next best equation was that of Bong et al. (2013) with MARE $=0.096, R M S E=0.269$, $S I=0.12$ and $B I A S=0.008$. Thus, according to the results, the equation suggested by the MLP-DT (Figure 7) for estimating the $\mathrm{Fr}$ performs well and can be used as an alternative for design engineers to estimate the incipient motion of sediment.

\section{REFERENCES}

Ab Ghani, A., Salem, A.M., Abdullah, R., Yahaya, A.S., Zakaria, N.A., 1999. Incipient motion of sediment particles over loose deposited beds in a rigid rectangular channel. Proc. $8^{\text {th }}$ Int. Conf. Urban Storm Drainage, Sydney, Australia.

Ab Ghani, A., Zakaria, N.A., Kassim, M., Nasir, B.A., 2001. Sediment size characteristics of urban drains in Malaysian cities. Urban Water J., 2, 335-341.

Ab Ghani, A., Azamathulla, H.M., Chang, C.K., Zakaria, N.A., Hasan, Z.A., 2011. Prediction of total bed material load for rivers in Malaysia: A case study of Langat, Muda and Kurau Rivers. Environ. Fluid Mech., 11, 3, 307-318.

Ackers, P., White, W.R., 1973. Sediment transport: new approach and analysis. J. Hydraul. Div., 99, 2041-2060. 
Ahmad, Z., Azamathulla, H.Md., Zakaria, N.A., 2011. ANFISbased approach for the estimation of transverse mixing coefficient. Water Sci. Technol., 63, 1005-1010.

Azamathulla, H.M., Ab Ghani, A., Zakaria, N.A., Kiat, C.C., Siang, L.C., 2008. Knowledge extraction from trained neural network scour models. Modern Appl. Sci., 2, 4, 52-62.

Bilhan, O., Emiroglu, M.E., Kisi, O., 2010. Application of two different neural network techniques to lateral outflow over rectangular side weirs located on a straight channel. Adv. Eng. Softw., 41, 831-837.

Bonakdari, H., Ebtehaj, I., 2014. Study of sediment transport using soft computing technique. In: Schleiss et al. (Eds): River Flow 2014, Chapter 116, pp. 933-940, Taylor \& Francis Group, London, UK.

Bong, C.H.J., Lau, T.L., Ab Ghani, A., 2013. Verification of equations for incipient motion studies for rigid rectangular channel. Water Sci. Technol., 67, 395-403.

Breiman, L., Friedman, J.H., Olshen, R.A., Stone, C.J., 1993. Classification and Regression Trees. Wadsworth, Inc. California, USA, 368 p.

Christopher, M., 1995. Neural networks for pattern recognition. Oxford University Press, Oxford, UK, 482 p.

Ebtehaj, I., Bonakdari, H., 2013. Evaluation of sediment transport in sewer using artificial neural network. Eng. Appl. Comput. Fluid Mech., 7, 382-392.

Ebtehaj, I., Bonakdari, H., 2014. Comparison of genetic algorithm and imperialist competitive algorithms in predicting bed load transport in clean pipe. Water Sci. Technol., 70, 1695-1701.

Ebtehaj, I., Bonakdari, H., 2016. Bed load sediment transport estimation in a clean pipe using multilayer perceptron with different training algorithms. KSCE J. Civil Eng., 20, 581589.

Ebtehaj, I., Bonakdari, H., Sharifi, A., 2014. Design criteria for sediment transport in sewers based on self-cleansing concept. J. Zhejiang-Univ. Sci-A., 15, 914-924.

Ebtehaj, I., Bonakdari, H., Khoshbin, F., Azimi, H., 2015. Pareto genetic design of GMDH-type neural network for predict discharge coefficient in rectangular side orifices. Flow Meas. Instrum., 41, 67-74.

El-Zaemey, A.K.S., 1991. Sediment transport over deposited beds in sewers. PhD Thesis, Newcastle University, Newcastle Upon Tyne, UK.

Gocić, M., Motamedi, S., Shamshirband, S., Petković, D., Ch, S., Hashim, R., Arif, M., 2015. Soft computing approaches for forecasting reference evapotranspiration. Comput. Electron. Agr., 113, 164-173.

Haddadchi, A., Movahedi, N., Vahidi, E., Omid, M.H., Dehghani, A.A., 2013. Evaluation of suspended load transport rate using transport formulas and artificial neural network models (Case study: Chelchay Catchment). J. Hydrodynamics, Ser. B., 25, 459-470.
Hagan, M.T., Demuth, H.B., Mark, H., Beale, M.H., 1996. Neural Network Design. PWS Publishing Company (Open Library), Boston, USA, $1012 \mathrm{p}$.

Haykin, S., 1994. Neural Networks, a Comprehensive Foundation. Practice Hall, New Jersey, USA, 823 p.

Ho, W.H., Tsai, J.T., Lin, B.T., Chou, J.H., 2009. Adaptive network-based fuzzy inference system for prediction of surface roughness in end milling process using hybrid Taguchigenetic learning algorithm. Expert Syst., Appl., 36, 32163222.

Kavousi-Fard, A., Kavousi-Fard, F., 2013. A new hybrid correction method for short-term load forecasting based on ARIMA, SVR and CSA. J. Exp. Theor. Artif. Intel., 25, 559-574.

Kisi, O., 2008. The potential of different ANN techniques in evapotranspiration modelling. Hydrol. Process., 22, 24492460 .

Kizilöz, B., Çevik, E., Aydoğan, B., 2015. Estimation of scour around submarine pipelines with Artificial Neural Network. Appl. Ocean Res., 51, 241-251.

Najafzadeh, M., Lim, S.Y., 2014. Application of improved neuro-fuzzy GMDH to predict scour depth at sluice gates. Earth Sci. Inform., 8, 187-196.

Najafzadeh, M., Barani, G.A., 2011. Comparison of group method of data handling based genetic programming and back propagation systems to predict scour depth around bridge piers. Sci. Iran., 18, 1207-1213.

Novak, P., Nalluri, C., 1984. Incipient motion of sediment particles over fixed beds. J. Hydraul. Res., 22, 181-197.

Oliver, N., 2001. Nonlinear System Identification: From Classical Approaches to Neural Networks and Fuzzy Models. Springer-Verlag, Berlin Heidelberg, Berlin, Germany, 785 p.

Safari, M.J.S., Mohammadi, M., Manafpour, M., 2011. Incipient motion and deposition of sediment in rigid boundary channels. In: Proc. $15^{\text {th }}$ Int. Conf. Transport \& Sedimentation of Solid Particles. Wroclaw, Poland.

Salem, A.M., 2013. The effects of the sediment bed thickness on the incipient motion of particles in a rigid rectangular channel. In: Proc. $17^{\text {th }}$ Int. Water Technology Conf., IWTC17, Istanbul, Turkey.

Shvidchenko, A.B., Pender, G., 2000. Flume study of the effect of relative depth on the incipient motion of coarse uniform sediments. Water Resour. Res., 36, 619-628.

Sun, S., Yan, H., Kouyi, G.L., 2014. Artificial neural network modelling in simulation of complex flow at open channel junctions based on large data sets. Environ. Model. Softw., 62, 178-187.

Vongvisessomjai, N., Tingsanchali, T., Babel, M.S., 2010. Non-deposition design criteria for sewers with part-full flow. Urban Water J., 7, 61-77.

Yalin, M.S., 1977. Mechanics of Sediment Transport. Pergamon Press, Oxford, UK, 360 p.

Received 16 October 2015 Accepted 10 May 2016 